\title{
Reflets
}

Revue ontaroise d'intervention sociale et communautaire

\section{Répondre aux besoins des travailleuses du sexe de rue : un objectif qui passe par la décriminalisation de leurs activités de travail}

\section{Colette Parent et Christine Bruckert}

Volume 11, numéro 1, 2005

Exclusion sociale

URI : https://id.erudit.org/iderudit/013061ar

DOI : https://doi.org/10.7202/013061ar

Aller au sommaire du numéro

Éditeur(s)

Reflets : Revue ontaroise d'intervention sociale et communautaire

ISSN

1203-4576 (imprimé)

1712-8498 (numérique)

Découvrir la revue

Citer cet article

Parent, C. \& Bruckert, C. (2005). Répondre aux besoins des travailleuses du sexe de rue : un objectif qui passe par la décriminalisation de leurs activités de travail. Reflets, 11(1), 112-145. https://doi.org/10.7202/013061ar

Tous droits réservés (C) Reflets : Revue ontaroise d'intervention sociale et communautaire, 2005
Ce document est protégé par la loi sur le droit d'auteur. L'utilisation des services d'Érudit (y compris la reproduction) est assujettie à sa politique d'utilisation que vous pouvez consulter en ligne.

https://apropos.erudit.org/fr/usagers/politique-dutilisation/ 


\section{Répondre aux besoins des travailleuses du sexe de rue : un objectif qui passe par la décriminalisation de leurs activités de travail}

\section{Colette Parent}

Professeure agrégée, département de criminologie, Université d'Ottawa

\section{Christine Bruckert}

Professeure adjointe, département de criminologie, Université d'Ottawa

La littérature sur le travail du sexe est aujourd'hui fort abondante. Les chercheures, analystes et militantes abordent tour à tour des dimensions théoriques de la question, documentent empiriquement les activités des différentes formes de pratiques, se penchent sur les composantes du travail qui peuvent affecter la santé des travailleuses, soumettent à la critique différentes politiques criminelles mises en œuvre et proposent des solutions. Mais les recherches sur les besoins des travailleuses du sexe, sur les services offerts et sur ceux qui seraient susceptibles de répondre de façon compréhensive à leurs besoins sont encore peu nombreuses. Cette situation apparait d'autant plus paradoxale qu'au premier regard, leurs besoins s'avèrent considérables, surtout en ce qui concerne les travailleuses du sexe de rue.

C'est dans ce contexte que le Centre Espoir Sophie, un centre de jour pour femmes marginalisées de la région de l'Outaouais, a exprimé l'intérêt d'une recherche sur les besoins des travailleuses du sexe de rue francophones, les ressources disponibles et celles à 
privilégier pour répondre de façon compréhensive à ces besoins. Les auteures de cet article ont alors formé un partenariat avec le Centre Espoir Sophie et élaboré une recherche-action ${ }^{1}$ sur les besoins des travailleuses du sexe de rue de la région et, plus spécifiquement, sur les travailleuses anglophones et francoontariennes à Ottawa et sur les travailleuses québécoises à Gatineau (secteur Hull).

Cette recherche-action a été menée avec l'appui d'un comité encadreur composé de représentantes de différents organismes communautaires de la région concernés par le problème et a pris appui sur les paramètres suivants : 1) la reconnaissance du travail du sexe comme un métier légitime et des droits des travailleuses du sexe d'une part et la prise en compte des besoins individuels des travailleuses du sexe dans l'exercice quotidien de leur métier d'autre part;2) un cadre théorique général qui aborde la "prostitution" comme une forme de travail et qui demeure attentif aux dimensions particulières des activités exercées à partir de la rue; 3) une démarche qui prend appui d'abord et avant tout sur la parole des travailleuses du sexe de rue avec qui cette recherche a été réalisée.

Dans les sections qui suivent, nous exposons d'abord les grandes lignes de la revue de littérature, notre cadre théorique et l'approche méthodologique que nous avons privilégiés. Ensuite, nous présentons les participantes puis nous nous penchons sur les besoins et services tels qu'elles ont exprimés.

\section{Une littérature qui illustre bien la controverse autour de la "prostitution »}

Comme signalé en introduction, les recherches sur les besoins des travailleuses du sexe, sur les services offerts et sur ceux qui seraient susceptibles de répondre de façon compréhensive à leurs besoins sont encore peu nombreuses. Quels sont les éléments qui ont mené à cette lacune? 
"...les recherches sur les besoins des travailleuses $d u$ sexe, sur les services offerts et sur ceux qui seraient susceptibles de répondre de façon compréhensive à leurs besoins sont encore peu nombreuses. "

"...c'est à travers les documents produits par les groupes de défense des droits des personnes prostituées que les travailleuses du sexe ont exprimé publiquement leurs besoins."
D'abord, comme certaines pratiques associées au travail du sexe sont illégales tant au Canada que dans d'autres pays occidentaux, notons que ces travailleuses sont d'une façon générale fort discrètes sur leurs pratiques de travail, peu faciles à rejoindre et ne participent à des recherches que lorsqu'elles sont rassurées sur les objectifs et intentions des chercheurs. Aussi, les recherches empiriques sont-elles parfois difficiles à mener; mais cette considération ne nous semble pas la plus déterminante pour expliquer l'état des études sur cette question.

En effet, pour nombre de chercheures, féministes ou non, le travail du sexe est fondamentalement associé à la victimation des femmes; elles envisagent alors les besoins des travailleuses dans la perspective de leur permettre de quitter ce travail. Cette vision, on le comprend, circonscrit la conception des besoins et des services aptes à y répondre. Vanwesenbeek (1994:10) souligne fort justement que lorsqu'on réfere à la question d'intervention en matière de "prostitution ", on se préoccupe rarement du bienêtre des travailleuses du sexe.

Par ailleurs, nous notons que les analyses produites à partir de sources gouvernementales puisent à des témoignages des différents groupes d'acteurs sociaux (policiers, résidents, représentantes de travailleuses du sexe) et inscrivent les besoins des travailleuses du sexe dans un contexte de régulation juridique et sociale. Aussi, si les solutions sont articulées en fonction de certains besoins des travailleuses du sexe, elles s'appuient essentiellement sur une conception du travail du sexe comme problème social et sur les préoccupations des autorités en matière d'ordre public. On tente donc de répondre aux besoins des travailleuses du sexe, ou du moins à ce que l'on en a retenu, sans mettre en cause la criminalisation des activités associées à leur travail. Dans ce cadre, l'expérience de ces travailleuses ne constitue qu'un élément d'analyse parmi d'autres.

Dans cette conjoncture peu propice à la réalisation de recherches empiriques basées sur leur témoignage, c'est à travers les documents produits par les groupes de défense des droits des personnes prostituées que les travailleuses du sexe ont exprimé publiquement leurs besoins. À cette production 
s'ajoutent certaines recherches contemporaines qui partent de la reconnaissance des travailleurs et travailleuses de l'industrie du sexe comme une autre catégorie professionnelle, avec des problèmes et des besoins spécifiques. De plus en plus, les groupes d'activistes et les féministes semblent se mettre d'accord sur ce point (Bullough et Bullough 1998).

Par ailleurs, comme le souligne Pryen (1999 :58), le contexte nouveau lié à l'émergence du SIDA a entraîné, à la fin des années 80 et dans les années 90, une reformulation de la problématique et des paramètres du débat sur la prostitution. En effet, l'urgence du problème et ses répercussions potentielles pour l'ensemble de la population, ont incité les différents acteurs sociaux à prendre en compte "les conditions de vie des personnes prostituées et les conditions d'exercice de leur activité dans une optique de prévention des risques et de prise en charge globale de la santé " (Pryen 1999 : 58). Malgré toutes les critiques qui peuvent être adressées à cette formulation du débat sur le travail du sexe, il est indéniable qu'elle a mené à l'implication des travailleuses du sexe elles-mêmes et à des partenariats mettant à contribution des acteurs sociaux de divers milieux, travailleurs sociaux, sociologues, épidémiologistes, travailleurs et travailleuses du sexe. Elle a également favorisé la réalisation d'études qui tiennent compte du bien-être des travailleuses du sexe.

Ces différentes publications, et en particulier celles qui émanent des organismes de défense des travailleuses du sexe, constituent donc les principales sources de cette revue de littérature qui porte, d'un côté, sur les besoins de reconnaissance du travail du sexe comme un métier légitime, de l'autre, sur les besoins individuels de ces travailleuses dans l'exercice de leur métier. De l'un à l'autre, on notera bien sûr des liens et des points de croisements et la nécessité de prendre en compte les uns pour pouvoir aborder les autres.

\section{La reconnaissance du travail du sexe comme métier}

C'est d'abord sur les plans juridique et politique que les représentantes des travailleuses du sexe ont identifié leurs besoins et proposé des solutions, l'objectif étant d'assurer l'inscription du 
"C'est d'abord sur les plans juridique et politique que les représentantes des travailleuses $d u$ sexe ont identifié leurs besoins et proposé des solutions, l'objectif étant d'assurer l'inscription $d u$ travail du sexe comme travail légitime dans le secteur des services, bénéficiant dès lors des protections qu'on accorde généralement à d'autres métiers. " travail du sexe comme travail légitime dans le secteur des services, bénéficiant dès lors des protections qu'on accorde généralement à d'autres métiers. Les déclarations de l'International Committee for Prostitutes' Rights (ICPR), issues du Second World Whores' Congress qui s'est tenu à Bruxelles en 1986, résument bien la position des regroupements des travailleuses du sexe. L'analyse dans son ensemble aussi bien que les remèdes proposés sont toujours d'actualité : après avoir énuméré une longue liste de violations de leurs droits, on propose que les travailleuses du sexe, et les ex-travailleuses, se voient accorder les mêmes droits que tous les autres citoyens. On propose aussi que la prostitution ait statut de profession légitime et que les travailleuses du sexe soient définies comme des citoyennes à part entière.

Au Canada, un certain nombre de ressources communautaires et d'organismes à but non lucratif ont mis au centre de leur philosophie et de leur action les besoins spécifiques des travailleuses du sexe. C'est le cas d'organismes comme Stella, Maggie's ou Pace qui se sont donné comme mandat, entre autres, de lutter contre la discrimination envers les travailleuses du sexe et de sensibiliser la population et les intervenants aux réalités de leur travail, dans un effort pour promouvoir l'adoption de politiques publiques mieux fondées et plus responsables.

En plus, le ICPR s'est penché sur les besoins spécifiques des travailleuses du sexe dans l'exercice au quotidien de leur métier. Nous exposons leur position ainsi que les résultats des recherches menées par des universitaires et des militantes sur cette dimension du problème.

\section{Les besoins et services reliés à l'exercice quotidien du métier}

Les besoins reliés à l'exercice du métier touchent la santé, la sécurité, le logement, l'éducation aussi bien que la coordination des services et le soutien social.

D'abord, c'est sans étonnement que l'on constate que les questions reliées à la santé sont au centre des préoccupations 
"...on constate que

les professionnels

manquent de

formation en ce qui

concerne les besoins de

santé des travailleuses

du sexe; leurs pratiques

auprès de cette clientèle

peuvent être teintées

de préjugés et de

jugements moraux. " pour la majorité des acteurs sociaux impliqués. Dans le cadre du deuxième congrès international des travailleuses du sexe évoqué ci-dessus, on souligne les problèmes relatifs à leur santé. On met également en évidence que la stigmatisation sociale et juridique dont elles font l'objet freine leurs possibilités d'accès aux services de santé. En effet, d'un côté, ces travailleuses ont servi historiquement de boucs émissaires pour les infections transmises sexuellement; de l'autre, elles sont contraintes à exercer leur métier sans protection sanitaire adéquate et ont difficilement accès aux services de santé dont elles ont besoin.

Visant dès lors dans ses réponses les plans politique et juridique, l'ICPR propose l'abrogation des lois qui déterminent le médecin consultant des travailleuses et l'abolition des certificats médicaux obligatoires. Il demande qu'on leur donne accès à des services de santé de leur choix, en toute confidentialité et à une assurancesalaire pour cause de maladie. Pour assurer la protection de leur intégrité physique et psychologique, il insiste sur la nécessité de leur accorder le droit de travailler à l'intérieur, de faire de la publicité, de solliciter dans la rue en respectant les règlements de zonage, etc.

L'ICPR et nombre de chercheurs mettent également en évidence les effets délétères de la stigmatisation sur la prestation de services de santé auprès des travailleuses du sexe. D'un côté, on constate que les professionnels manquent de formation en ce qui concerne les besoins de santé des travailleuses du sexe; leurs pratiques auprès de cette clientèle peuvent être teintées de préjugés et de jugements moraux. Guénette et Léveillé (2002) ont constaté, par exemple, que bien que plusieurs travailleuses du sexe du Centre-Ville de Hull (Gatineau) aient fréquenté la clinique ambulatoire du Centre local de services communautaires, certaines semblent ne pas vouloir utiliser à nouveau leurs services parce qu'elles y auraient été mal accueillies.

En réponse à ce problème, l'ICPR propose que l'on forme et éduque les professionnels sur les problèmes de santé reliés au travail du sexe de façon à combattre les préjugés et les connaissances erronées qui circulent dans le milieu médical, que l'on assure la participation de travailleuses du sexe et d'ex-travailleuses du sexe 
aux séances d'information et que l'on intègre obligatoirement des travailleuses du sexe dans les effectifs de santé afin de favoriser l'adoption de politiques publiques appropriées et la mise en oeuvre de services adéquats.

Par ailleurs, les travailleuses du sexe ont des besoins de santé spécifiques. Bien qu'elles ne soient pas plus à risque d'être victimes d'infections transmises sexuellement (ITS) que d'autres groupes dans la population (Pheterson 1989; Gibson 1993; Welzer-Lang, Barbosa, Mathieu 1994), elles sont sexuellement actives et doivent se prémunir contre ces infections, en particulier le SIDA. Du point de vue des groupes militants, le plus grand risque en cette matière est celui de la contamination des travailleurs et travailleuses du sexe par les clients. Plusieurs stratégies sont alors proposées, en particulier, l'usage systématique des préservatifs. Certains favorisent des ateliers sur les pratiques sexuelles sécuritaires (safe sex workshops) pour les travailleuses du sexe (Gibson 1993 : 174). Aux Pays-Bas, ces dernières portent une étiquette gommée ou une affiche pour indiquer qu'elles s'engagent seulement dans des pratiques sexuelles sécuritaires (Sterk-Elifson, Campbell 1993 : 201).

Par ailleurs, les problèmes de toxicomanie font aussi l'objet de préoccupations, auprès surtout des travailleuses du sexe de rue qui, selon l'ensemble des recherches, consomment des drogues dans un pourcentage manifestement plus élevé que les autres catégories de travailleuses du sexe. ${ }^{2}$

En France, le comité AIDES Nord-Pas-de-Calais, un organisme de terrain, a adopté l'approche de la réduction des méfaits et propose des services directement sur les lieux de travail, soit la rue, à des heures variées et avancées dans la nuit. Des unités mobiles stationnent de façon régulière dans les quartiers et offrent du matériel d'injection stérile (comme des seringues, des tampons alcoolisés, de l'eau stérile, de l'acide citrique), des préservatifs, du café et des gâteaux. Ce sont des lieux de sociabilité : quand le «bus " s'installe dans les quartiers de prostitution, ce sont surtout des travailleuses du sexe, usagères de drogues ou non, qui bénéficient de ses services (Pryen 1999 :61). Également en France, une association composée à parité de professionnels de la santé, de travailleuses du sexe et de chercheurs, Cabiria, a mis sur pied un 
projet de santé communautaire sur les territoires de la prostitution lyonnaise. En s'inspirant d'une approche globale de la santé, ce projet favorise d'abord et avant tout les actions sur le terrain telles les sorties de nuit avec des infirmiers ou des intervenants en prévention. Il offre également des services diversifiés en réponse aux besoins globaux des travailleuses du sexe : prévention et soins en matière de santé et, par la suite, aide et orientation sur des questions sociales et administratives de même qu'en matière de droits humains et de citoyenneté. Il met de plus l'accent sur la formation d'un réseau de partenaires ressources, constamment réactualisé aux besoins des travailleurs et travailleuses du sexe ainsi que sur la "coordination avec des organismes ou des groupes existants, faisant un travail identique ${ }^{3}{ }^{3}$

La sécurité renvoie également à un aspect important des besoins des travailleuses du sexe, principalement celles qui oeuvrent dans la rue. Les recherches démontrent que ces dernières sont plus exposées à la violence que les autres travailleuses du sexe. Elles peuvent être la cible des clients, des agresseurs; celle aussi des souteneurs, de la police et des membres de la communauté. En 1997, le Centre canadien de la statistique juridique a estimé que « 63 personnes connues pour se livrer à la prostitution ont été assassinées entre 1991 et 1995. Presque toutes les victimes étaient des femmes (60 des 63) » (Ministère de la Justice du Canada 1998 : 4 Partie I). Il faut aussi souligner les abus d'autorité des forces policières dont les travailleuses du sexe de rue font plus souvent l'objet que les autres travailleurs du sexe. Guénette et Léveillé (2002) rapportent de nombreuses plaintes de harcèlement policier envers les travailleuses du sexe dans le Centre-Ville de Gatineau (Hull).

Les mesures proposées pour répondre à ce besoin varient selon qu'elles émanent des autorités gouvernementales ou des associations des travailleuses du sexe. Les premières cherchent des solutions qui satisfont à la fois les préoccupations d'ordre public et celles qui entourent la protection des travailleuses du sexe. Ne présentant pas, selon elles, de véritables solutions à la marginalisation, les représentantes des organismes de défense des travailleuses du sexe endossent rarement ces mesures. Par contre, 
en réponse immédiate à ce problème pressant, ces porte-parole en proposent d'autres, dont l'élaboration et le maintien à jour d'une liste de mauvais clients.

Les chercheurs mettent en évidence un autre besoin des travailleuses du sexe de rue, soit la quête d'un logement à prix modique où elles trouveraient sans délai un refuge sécuritaire (Rabinovich, Strega 2004; Dalla, 2002; Benoit, Millar 2001; Pyett, Warr 1999, Benson, Matthews 1995; Lowman 1986-87). Thukral et Ditmore $(2003: 10)$ proposent la création de maisons d'hébergement pour travailleuses du sexe de rue à New York dont le concept ressemblerait à celui des maisons pour conjointes victimes de violence. Dans ces refuges temporaires pour les travailleuses, les intervenantes auraient pour mandat, entre autres, de les assister dans la recherche d'un logement et de les aider à y emménager.

Plusieurs auteurs signalent également les besoins des travailleuses du sexe en matière d'éducation, de formation à l'emploi (Surrat et al. 2004; Dalla 2002; Norton-Hawk 2001; Benson et Matthews 1995; Lowman 1985-86). Une étude menée à Ottawa sur le travail du sexe de rue indique que les femmes ne font pas de recherches d'emplois réguliers et que lorsque cela se produit, celles-ci n'aboutissent pas, le plus souvent à cause de leur manque d'instruction (Ministère de la Justice du Canada1998: 18 Partie III).

Certains programmes de formation sont donc mis sur pied et offerts aux travailleuses du sexe qui désirent changer de métier. Par exemple, l' Option Youth Society deVancouver a ouvert un restaurant en 1989 et un centre de formation pour les jeunes prostitués de rue qui voulaient travailler dans le secteur hôtelier. Ils ont reçu une formation et un encadrement social en plus de bénéficier d'un service de placement. L'initiative a permis l'ouverture du café Picasso.

Il faut également souligner que, compte tenu de leur stigmatisation et de leur marginalisation, les travailleuses du sexe, en particulier celles qui travaillent dans la rue, ont non seulement besoin de services, mais aussi de mesures de coordination pour 
"...les recherches empiriques mettent en lumière le manque de services sociaux adaptés aux besoins des travailleurs et travailleuses du sexe de rue."

"On se doit de favoriser des modes d'action communautaires, paritaires, qui mettent l'accent sur le respect, l'entraide et le soutien des personnes impliquées dans le travail du sexe." avoir accès aux services. Pour briser leur isolement, il faut aussi prévoir des lieux de sociabilité et des initiatives de soutien social.

En effet, les recherches empiriques mettent en lumière le manque de services sociaux adaptés aux besoins des travailleurs et travailleuses du sexe de rue. On sait que les travailleuses du sexe ne peuvent pas recourir à certains services s'adressant à la population en général, soit parce qu'elles en ignorent l'existence, soit en raison de difficultés liées à leur utilisation, par exemple, les heures d'ouverture, soit pour des motifs d'ordre culturel ou social, tel le fait de ne pas maittriser la langue dans laquelle le service est offert. Notons également que les travailleuses du sexe se sentent jugées par des intervenants du secteur médical ou des policiers qui, le plus souvent, n'ont pas reçu une formation adéquate pour prendre en compte leurs besoins spécifiques (Ministère de la Justice du Canada 1998 : 16 Partie III).

Pour répondre à ce problème, plusieurs auteurs mentionnent la nécessité d'une approche globale inscrite dans la perspective de la réalisation complète de la citoyenneté des travailleuses du sexe (Pryen 1999). Celle-ci constituerait une réponse aux approches restreintes en termes de santé publique, d'ordre public ou d'enjeu moral. C'est donc dire que pour réorienter l'intervention à partir des besoins des travailleuses du sexe, il faut se demander, comme le suggère Pryen (1999 : 51), quels sont « les principaux rapports aux institutions que vivent les personnes prostituées ". À quel type de logiques ces personnes sont-elles exposées? Il faut dès lors repenser le paysage institutionnel et associatif pour l'adapter aux réalités concrètes du travail du sexe de rue. Selon le Ministère de la Justice du Canada (1998), il faudrait assurer une meilleure coordination entre les politiques et les organismes tant étatiques que communautaires et associatifs. On se doit de favoriser des modes d'action communautaires, paritaires, qui mettent l'accent sur le respect, l'entraide et le soutien des personnes impliquées dans le travail du sexe.

Pour rejoindre les travailleuses du sexe de rue, les actions de terrain, sur le trottoir, sont tout indiquées. Les unités mobiles d'accueil représentent en effet une stratégie fort intéressante dans la 
mesure où elles répondent à des paramètres essentiels de flexibilité, de proximité et de mobilité. Les intervenantes peuvent y distribuer du matériel pédagogique, offrir du soutien psychologique, fournir des conseils, etc. Les travailleuses du sexe de rue ont aussi l'occasion d'y prendre une pause, de consommer un thé ou un café, de parler de leurs problèmes, besoins ou expériences, d'échanger avec d'autres, de faire provision de préservatifs, seringues ou autres matériaux nécessaires pour travailler dans de meilleures conditions. En Europe, les « boutiques » offrent le même type de services dans des zones de prostitution de rue, sur les lieux de l'action. Notons que l'existence de ces lieux d'accueil ${ }^{4}$ sur le site même du travail améliore la sécurité des travailleuses du sexe de rue et constitue une mesure contre leur isolement.

Selon la revue de littérature, les centres de jour représentent des ressources précieuses pour les travailleuses du sexe de rue. Ils constituent des lieux de repli, d'accueil et d'écoute qui répondent à une pluralité de leurs besoins, les uns plus pragmatiques, les autres plus liés à leur bien-être émotionnel et psychologique. Comme le soulignent Guénette et Léveillé (2002), il faut permettre aux travailleuses du sexe de se rassembler, de créer un réseau de soutien adapté à leur culture. Par exemple, à Vancouver, le Wish Drop-In Centre Society (http://www.wish-vancouver.net) est un organisme ayant pour mandat de répondre directement aux besoins quotidiens et pragmatiques des travailleuses du sexe de rue. Dans ce centre de jour, de nombreux produits et services sont offerts : des repas chauds, des vêtements, du maquillage, des douches. On peut aussi compter sur des soins infirmiers, un service d'hébergement pour les travailleuses du sexe dans le besoin, des programmes de transition pour des femmes à la recherche d'un emploi dans un autre domaine et des programmes d'alphabétisation. On réfere, au besoin, vers d'autres services sociaux spécialisés, tels des centres de désintoxication ou de réhabilitation. Certains centres de jour produisent des listes de mauvais clients pour améliorer la sécurité des travailleuses du sexe. On y fait la promotion de comportements sexuels sécuritaires et la distribution de préservatifs, de seringues et de lubrifiants. 
Dans l'ensemble, ce type de services répond aux besoins quotidiens des travailleuses du sexe de rue sans faire l'impasse sur la stigmatisation pénale et sociale qui structure en partie leurs besoins, leur capacité d'accéder aux services disponibles et à un réseau non officiel de soutien.

En résumé, la littérature met en évidence les différents types de besoins des travailleuses du sexe et en souligne l'importance pour celles qui œuvrent dans la rue. Elle pave la voie pour aborder cette question dans le cadre plus spécifique des travailleuses du sexe de rue de la région d'Ottawa-Gatineau.

\section{Un cadre théorique qui prend appui sur la "prostitution» comme travail}

"Nos réflexions théoriques et nos études empiriques nous ont, en effet, amenées à définir la " prostitution » comme un métier d'abord et avant tout féminin et, plus précisément, comme un métier non institutionnalisé dans le domaine des services. »
Dans le cadre de cette recherche, comme dans d'autres études que nous avons menées précédemment, nous avons observé les activités des participantes en tant qu'activités de travail. Nos réflexions théoriques et nos études empiriques nous ont, en effet, amenées à définir la "prostitution " comme un métier d'abord et avant tout féminin et, plus précisément, comme un métier non institutionnalisé dans le domaine des services. Ce métier met en cause l'association dominante entre sexe et intimité (Parent et Bruckert 2005). Au Canada, il est souvent exercé par des travailleuses autonomes ou alors, dans le cadre d'une petite organisation ne regroupant que quelques personnes. L'organisation du travail et les activités de travail varient selon les lieux ou les formes de pratiques (salons de massage, établissements érotiques, escortes, travail du sexe de rue). Un certain nombre d'activités reliées à ce travail sont considérées comme illégales et, dans l'ensemble, les travailleuses du sexe font l'objet de stigmatisation. Cependant, les recherches suggèrent que le travail du sexe de rue expose davantage les femmes à la violence et à l'intervention des forces de l'ordre.

Pour considérer les pratiques de prostitution comme des activités de travail, il nous semble qu'elles doivent répondre à un 
certain nombre de conditions :1) elles doivent cibler des échanges consensuels de services entre adultes consentants contre une forme de rémunération; 2) elles doivent remplir des conditions plus ou moins nombreuses et souples d'organisation, c.-à-d. cibler un éventail de services, offerts dans un cadre de travail plus ou moins stable (lieu, journées, heures, relations avec collègues, clients, etc.);3) elles doivent, essentiellement, être associées à une autoreprésentation comme travailleuse.

Or, bien que plusieurs recherches indiquent qu'un nombre important de travailleuses du sexe de rue considèrent explicitement leurs pratiques comme un métier (Pryen 1999, McKeganey et Barnard 1996), nous avons relevé deux caractéristiques parfois associées au travail du sexe de rue qui posent question quant à l'approche de ces pratiques comme forme de travail. Des auteurs désignent certaines formes de prostitution de rue comme des pratiques de survie (survival sex). D'autres mettent l'accent sur la consommation de drogues qui apparait particulièrement répandue chez les travailleuses du sexe de rue et qui amène à se demander comment la dépendance des drogues peut affecter le rapport au travail de ces femmes. Nous discutons tour à tour de ces deux points.

Lorsque nous examinons la littérature qui réfere aux services de survie (survival sex), nous notons que les auteurs visent le plus souvent une clientèle de jeunes, des mineurs, et situent leurs recherches dans des pays fortement marqués par la pauvreté, le dénuement et le sida. Ils évoquent plutôt des activités ponctuelles, qu'elles soient fréquentes ou sporadiques, qui représentent une sorte d'expédient, un moyen parmi d'autres pour se procurer de la nourriture, un toit pour la nuit, des vêtements (Lockhart 2002; Preston-White 1996; Richter et Swart-Kruger 1995). Certains auteurs ont importé le concept au Canada pour référer principalement aux services sexuels des mineurs, mais aussi des jeunes adultes. On note l'inclusion de la consommation de drogues comme facteur relié à ces comportements (Spittal et al 2003; Tyler et al. 2000). Il y a alors association entre service sexuel rendu dans une perspective de survie et celui qui est offert pour répondre à un manque de drogues dont l'organisme dépend et qu'il réclame 
impérativement. En ce qui nous concerne, c.-à-d. au niveau du travail du sexe des adultes, l'usage du concept de survival sex apparait problématique. Il fait l'impasse sur l'observation des activités de prostitution comme une forme de travail non institutionnalisé et sur les liens complexes et potentiellement paradoxaux entre travail et consommation de drogues. La littérature sur les liens entre drogues et travail du sexe confirme notre appréhension du problème.

En effet, lorsque nous considérons les études des années 80 sur le travail du sexe et la consommation d'héroïne, celles des années 90 sur le travail du sexe et la consommation de cocaïne, en particulier sous la forme du crack. et que nous y intégrons les données issues de nos propres recherches empiriques, le portrait d'ensemble nous apparait beaucoup plus complexe.

D'abord, nous retrouvons des travailleuses du sexe consommatrices de drogues qui définissent leurs activités comme des activités professionnelles et qui évaluent le travail à partir de certains critères d'organisation et la qualité des services rendus. Elles considèrent qu'il est important de maintenir en tout temps un rapport professionnel avec les clients, indépendamment de leur consommation. Rosenbaum (1981) précise que, pour les travailleuses du sexe héroïnomanes qu'elle a interviewées, cela signifie maintenir une routine, une bonne apparence, négocier les prix et rendre adéquatement les services. La dépendance de drogues est donc perçue comme un problème qui peut affecter leur capacité de maintenir un rapport professionnel au travail. Blom et Van den Berg (1989) en font également état lorsqu'ils étudient la consommation d'héroïne chez les travailleuses du sexe. Plusieurs participantes à notre recherche sur le travail du sexe dans les salons de massage et les établissements érotiques ont affirmé que la consommation de drogues représentait, quant à elles, un problème potentiel au niveau du travail et non une solution (Parent et Bruckert 2005).

D'un autre côté, plusieurs auteurs qui se sont penchés sur la consommation du crack (Boyle et Anglin 1993; Inciardi, Lockwood, Pottieger 1993; Koester et Schwart 1993; Sterk 1999 etc.), indiquent que les travailleuses du sexe qui commencent à 
consommer cette drogue maintiennent la règle de se faire payer leurs services en argent et refusent le troc avec de la drogue. Koester et Schwartz (1993) précisent qu'en exigeant les paiements en argent, elles sont en mesure de négocier une entente de services dans le cadre de laquelle elles peuvent discuter et du prix et du type de prestations. Cet argent leur permet certes de se procurer de la drogue, mais aussi d'autres biens pour elles et leur famille. Ces travailleuses du sexe, qui se définissent d'ailleurs comme des professionnelles en opposition aux consommatrices de crack qui vendent des services sexuels directement pour de la drogue, peuvent également se payer des condoms. Les chercheurs notent chez elles une préoccupation soutenue pour des pratiques sexuelles sécuritaires.

Face à ce groupe de travailleuses du sexe qui consomment des drogues tout en maintenant une approche professionnelle aux services qu'elles offrent, on peut identifier des consommatrices de drogues qui vendent des services sexuels pour se procurer soit de l'héroïne, soit du crack. Ces dernières ne développent pas un rapport professionnel à la prostitution qu'elles conçoivent plutôt comme un expédient qui leur permet de se procurer de la drogue. Surtout depuis la mise en marché du crack, on constate que plusieurs acceptent de rendre des services sexuels directement pour de la drogue et perdent ainsi contrôle sur les paramètres de leurs prestations de services (prix et type de services). Selon Ratner (1993), l'échange de services sexuels pour de la drogue ou encore pour un montant d'argent dérisoire, caractérise des pratiques désespérées de personnes dont toute la vie est dominée par le besoin de drogues. Les auteurs évoquent l'extrême subordination dans laquelle se retrouvent ces femmes pour négocier le service à rendre.

En somme, pour les travailleuses du sexe qui consomment des drogues, rendre des services sexuels demeure un travail et elles luttent contre sa dévaluation alors que pour les autres, il ne s'agit que d'un expédient parmi d'autres pour obtenir des drogues. Bien sûr, ces deux positions n'épuisent pas les scénarios possibles touchant les rapports entre drogues et prostitution; elles n'en représentent que deux pôles. Mais, elles nous permettent 
d'appréhender la complexité de la question et indiquent que les besoins des travailleuses du sexe peuvent varier grandement selon leur rapport aux drogues et à leur métier.

\section{La méthode choisie : la recherche-action}

"Pour cerner la diversité des besoins des travailleuses $d u$ sexe et les services aptes à y répondre, nous avons inscrit notre démarche dans le cadre, diversifié, des recherches féministes qui reconnaissent d'entrée de jeu l'importance des rapports sociaux de genre dans toutes les sphères de la vie, $y$ compris dans le domaine de la production des connaissances. "
Pour cerner la diversité des besoins des travailleuses du sexe et les services aptes à y répondre, nous avons inscrit notre démarche dans le cadre, diversifié, des recherches féministes qui reconnaissent d'entrée de jeu l'importance des rapports sociaux de genre dans toutes les sphères de la vie, y compris dans le domaine de la production des connaissances. Nous avons privilégié ici la recherche-action: le problème a d'abord été identifié dans la communauté et c'est avec la participation de représentantes de cette communauté que nous avons amorcé la démarche de recherche. Par ailleurs, comme le soulignent Barnsley et Ellis (1992 : 9), la recherche-action met l'accent sur la cueillette systématique et l'analyse des données dans le but d'amener des changements. Nous nous sommes alors appuyées sur la parole des travailleuses du sexe, soucieuses de leur donner accès à l'espace discursif et de produire des connaissances qui puissent leur être utiles et se répercuter dans des programmes adaptés à leurs besoins.

Initialement, ce sont des participantes au Centre Espoir Sophie, un centre de jour pour femmes situé dans le secteur du marché By à Ottawa, qui ont fait état d'un manque de services adaptés à leurs besoins. Par ailleurs, des intervenantes avaient constaté que ce centre, ouvert en 1997 pour répondre aux besoins des travailleuses du sexe francophones de la région, comptait peu de travailleuses du sexe parmi sa clientèle. Elles avaient également noté que les ressources pensées pour ces travailleuses étaient en général plutôt limitées. La situation était d'autant plus complexe qu'on retrouve dans la région deux groupes linguistiques et deux juridictions provinciales différentes. On devait donc, d'un côté, considérer les besoins des travailleuses tant anglophones que francophones d'Ottawa, en Ontario, qui occupent trois territoires de travail, soit 
le secteur du marché By, le secteur deVanier et celui d'Hintonberg et, de l'autre côté, ceux des travailleuses francophones du Québec, qu'on retrouve dans le vieux Hull. Notons que les travailleuses se déplacent d'un territoire à l'autre, d'une province à l'autre, surtout, lorsque les policiers intensifient la surveillance dans un secteur.

À la suite de la formulation de ce problème, nous avons mis sur pied une recherche-action sur les besoins et services pour les travailleuses du sexe de rue dans les limites géographiques de la région d'Ottawa-Gatineau. Le premier objectif était d'identifier les besoins des travailleuses du sexe de rue, tant francophones qu'anglophones, et les services nécessaires pour y répondre. Le second était de répertorier les services disponibles, en français et en anglais, pour les travailleuses du sexe de la région. Une fois ces deux objectifs réalisés, nous voulions développer un plan d'action d'ensemble pour améliorer les ressources et services disponibles pour les travailleuses du sexe. Enfin, nous voulions améliorer la sécurité des travailleuses du sexe en leur donnant des outils pour former un groupe de défense et pouvoir ainsi ajouter officiellement leur voix à l'ensemble des acteurs sociaux qui contribuent à définir la problématique du travail du sexe dans la région. Pour assurer la mise en œuvre du premier volet de ce projet, soit la recherche sur les besoins des travailleuses du sexe et les services nécessaires pour y répondre, nous avons formé un comité consultatif composé de six représentantes d'organismes francophones de la région et d'un membre de la communauté qui est en relation avec ces dernières.

Nous nous étions donné comme objectif d'interviewer 18 travailleuses du sexe, soit six Québécoises qui travaillent principalement dans le secteur Hull, six anglophones et six franco-ontariennes travaillant principalement dans les secteurs de la ville d'Ottawa. Nous avons privilégié un «échantillon par filière " ou par "boule de neige ». Le comité consultatif nous a servi de courroie de transmission pour établir les contacts avec les travailleuses du sexe. Les membres nous ont indiqué des intervenantes ou des membres de la communauté qui avaient des contacts avec des travailleuses du sexe, qui à leur tour, nous ont référées à des travailleuses. Par la suite, certaines travailleuses nous 
ont mises en contact avec des collègues de travail. Nous avons finalement pu réaliser 19 entrevues, soit sept avec des travailleuses du sexe franco-ontariennes, six avec des Anglo-Ontariennes et six avec des Québécoises.

Nous avions mis au point un protocole d'entrevue composé de deux grandes sections. Dans la première, nous avions ciblé différents aspects du travail (activités de travail, journée type, dimensions positives et négatives, conciliation vie sociale et travail); dans la seconde, nous avions posé des questions relatives à leurs connaissances des ressources disponibles, à leur accès et à la fréquentation de celles-ci; nous avions exploré leurs besoins et les services qui permettraient d'y répondre.

Nous avons procédé à l'analyse entrevue par entrevue et dégagé les thèmes majeurs. Ensuite, nous avons analysé les entrevues de chacun des groupes de notre échantillon (travailleuses anglophones, francophones de Hull, franco-ontariennes) pour faire ressortir leurs particularités. D'un groupe à l'autre, nous avons constaté peu de variation significative. Il est possible qu'il $\mathrm{y}$ en ait de fait très peu, mais il faut également reconnaître que les sous-groupes de notre échantillon étaient de taille limitée (de 6 à 7 participantes). Lorsqu'identifiées, les particularités qui nous apparaissaient avoir une valeur importante ont été prises en compte dans l'analyse. Nous avons finalement procédé à une analyse d'ensemble.

Le profil des participantes renvoie à un groupe de travailleuses fort diversifié au niveau de l'âge et comptant une expérience notable dans le travail du sexe de rue.

En effet, nous constatons que les participantes ont entre 24 et 43 ans et que la moyenne du groupe est de 37 ans. Contrairement à un préjugé assez répandu, ce type de métier n'est pas seulement réservé aux très jeunes femmes. Dans l'ensemble, le niveau d'éducation des participantes est peu élevé, la majorité n'ayant pas complété d'études secondaires. Par contre, presque la moitié d'entre elles présentent une expérience de travail dans un domaine autre que le travail du sexe et la plupart accumulent une bonne expérience du travail dans la rue; la moyenne est de 14 ans. 
"...la plupart d'entre elles reçoivent, ou recevaient durant une certaine période de leur vie, des prestations d'assistance sociale, ce qui illustre bien la vulnérabilité sociale de ce groupe des femmes contraintes de vivre aux marges de la société."
Au plan financier, le tableau d'ensemble indique également une grande diversité. On compte trois travailleuses gagnant moins de 10000 \$ par année alors que six rapportent des revenus annuels supérieurs à 40000 \$. Par contre, la plupart d'entre elles reçoivent, ou recevaient durant une certaine période de leur vie, des prestations d'assistance sociale, ce qui illustre bien la vulnérabilité sociale de ce groupe des femmes contraintes de vivre aux marges de la société. Cela nous amène également à anticiper que nombre d'entre elles peuvent partager des besoins avec d'autres couches défavorisées de la population. La présentation de résultats nous permettra d'étayer ce point.

\section{Le problème, les solutions du point de vue des travailleuses du sexe}

\section{Un métier et ses avantages}

À l'instar d'autres recherches sur le travail du sexe de rue (McKeganey et Barnard 1996; Pryen 1999), nous avons constaté que la grande majorité des participantes considèrent la prestation des services sexuels comme un travail en soi, bien qu'elles en font une évaluation qui varie beaucoup de l'une à l'autre.

On note d'emblée que toutes apprécient l'aspect financier du travail. Plusieurs soulignent également la flexibilité des horaires qui leur permet de bien coordonner leur travail et les exigences relatives à leur vie personnelle. Pour quelques-unes, la possibilité de développer de bonnes relations avec les clients constitue un aspect positif du travail. Smiley indique qu'elle a maintenu des relations avec des clients qui sont devenus des amis, qui l'appellent et qui lui viennent en aide à l'occasion lorsque sa situation financière est difficile. D'autres affirment puiser dans les services auprès de leur clientèle un soutien pour leur amour-propre, pour leur estime de soi.Tikiboo rapporte qu'elle se sent appréciée, valorisée par les clients avec qui elle entretient une relation mutuellement marquée par le respect. 
Mais, tout en reconnaissant que c'est un métier, certaines n'y apprécient que l'aspect financier. Marie, qui a été travailleuse du sexe de rue pendant deux ans, rapporte l'avoir fait essentiellement pour l'argent qui lui permettait d'acheter de la cocaïne. Pour elle, cette expérience a été un enfer, compte tenu, entre autres, des conditions de travail qu'impose la criminalisation des activités associées à la prostitution. L'entrevue de Marie révèle qu'elle n'a pas développé une approche professionnelle à son travail. Alexandra ne fait le travail du sexe de rue que pour compléter ses fins de mois et reconnaît que c'est un travail qui va à l'encontre de ses valeurs morales.

Enfin, pour certaines participantes, le travail du sexe est si étroitement associé à la consommation de drogues qu'elles ne le conçoivent pas comme un métier. Tout service sexuel est relié à la perspective de l'achat imminent de drogues, de sorte que les deux activités apparaissent solidement imbriquées l'une dans l'autre. Non seulement le travail, mais aussi les loisirs, sont pensés essentiellement sous le signe de la consommation des drogues. La vie de ces femmes s'y trouve complètement absorbée.

Nos données confirment donc que la conception du travail du sexe comme métier n'est pas actualisée chez toutes les travailleuses du sexe. Elles laissent entrevoir l'impact possible, mais variable de la consommation de drogues sur leur perception et elles indiquent que les valeurs morales constituent un autre paramètre susceptible d'affecter le regard des travailleuses. Pour appréhender de façon plus compréhensive cette question, il nous faudrait mener des entrevues dans une perspective de carrière en repérant les changements au niveau des représentations au fil des années d'expérience, du type de pratiques et du début ou de l'arrêt de consommation des drogues.

\section{Un métier sous interdits juridiques et sous risques de violence}

Nous savons que le travail du sexe n'est pas directement interdit au Canada, mais nombre d'activités qui y sont associées tombent sous la juridiction du système pénal. En vertu de l'article 213 du Code 
"...comme plusieurs dimensions de leur travail tombent sous le coup de la loi, les travailleuses du sexe sont fort vulnérables face aux clients et sont souvent victimes de leur violence. " criminel canadien, la communication aux fins de prostitution sur la voie publique est l'infraction pour laquelle on entame le plus grand nombre de poursuites. Comme les travailleuses du sexe de rue recrutent leurs clients d'abord et avant tout dans la rue, elles sont visibles et font l'objet de beaucoup de surveillance policière. Elles peuvent difficilement travailler sur une base régulière sans faire l'objet d'arrestations et d'accusations, en particulier pour communication.

Aussi, est-ce sans trop de surprises que 18 des 19 femmes interviewées ont rapporté avoir eu des ennuis avec la loi.En effet, la plupart ont été accusées en vertu de lois relatives à la prostitution et pour des bris de conditions de probation ou de libération conditionnelle. Plusieurs ont eu des problèmes juridiques répétés. Wendy constitue, à cet égard, un cas extrême; elle a été arrêtée plus de 80 fois et condamnée à 16 ou à 17 reprises. Signalons que quelques-unes ont aussi été prises en charge pour des activités reliées aux drogues.

Par ailleurs, comme plusieurs dimensions de leur travail tombent sous le coup de la loi, les travailleuses du sexe sont fort vulnérables face aux clients et sont souvent victimes de leur violence. Les recherches sur le travail du sexe de rue soulèvent systématiquement cet aspect. Celle que nous avons menée indique encore une fois l'ampleur de ce problème.

La presque totalité des participantes, soit seize d'entre elles, rapporte avoir subi de la violence de la part de clients. Cocotte avait accepté d'accompagner chez lui un client qui avait l'air respectueux et inoffensif. La suite des événements lui a donné tort :

"Quand j'ai été chez eux j'ai été enfermée pendant deux heures de temps avec le gun sur la tête. Ça été comme...J'ai été sauvée par un de ses amis, parce qu'un de ses amis est arrivé là (...) moi j'étais là, je braillais. Puis je lui dis : tu peux m'amener chez nous. Il dit oui (...).» 
"Pour éviter les arrestations, les travailleuses du sexe essaient de mener discrètement leurs activités, devenant, du même coup, moins visibles, ce qui accroît leur vulnérabilité. "

"...non seulement les policiers n'offrent pas de protection, mais certains ont eux-mêmes recours à la violence envers les travailleuses du sexe. »
Pour éviter les arrestations, les travailleuses du sexe essaient de mener discrètement leurs activités, devenant, du même coup, moins visibles, ce qui accroît leur vulnérabilité. Même lorsqu'elles sont victimes, le recours à la police ne constitue pas une solution : la seule travailleuse du sexe qui a passé outre à cette règle tacite s'est retrouvée elle-même accusée d'une infraction. En effet, Candy rapporte qu'un prétendu client l'a fait monter dans sa voiture puis devant l'impossibilité de conclure une entente, il a refusé de la laisser partir et s'est mis à la violenter :

"He won't let me go. He takes me down by the
marina, down by Hull. Rips the buttons right off my
dress, starts beating me up. Nothing sexual, he just
wants to hurt me. Beating me up. Finally somebody
calls the cops, they come. You know what? They arrest
me! For prostitution! They let him go! And I - no
bra or underwear, dress wide open, black and blue, blee-
ding and they arrested me! The Hull police.You know.
Because - and I told them, yeah I was working on
the Market and this guy picked me up and he's beaten
the shit out of me. Fucking arrest him! I got rights."

Plus encore, non seulement les policiers n'offrent pas de protection, mais certains ont eux-mêmes recours à la violence envers les travailleuses du sexe. Un nombre important d'entre elles, soit sept femmes sur dix-neuf, font état d'incidents avec la police, allant de l'agression verbale jusqu'aux coups.Venant d'être brutalisée par un client et s'enfuyant à moitié nue vers la route Naomi nous rapporte qu'à leur arrivée, les policiers l'ont traitée avec beaucoup de mépris lorsqu'ils ont constaté qu'elle était une travailleuse du sexe.

Quelques-unes ont aussi eu des incidents avec la communauté. Certaines participantes nous ont confié que des citoyens les couvraient d'insultes quand elles déambulaient dans la rue. Pour Wendy, une transsexuelle, les problèmes sont encore plus sérieux. Elle a indiqué en entrevue que des individus l'insultaient et lui lançaient des objets lorsqu'elle était sur la place publique. Cocotte signale de son côté que des voisins l'enjoignaient à aller travailler ailleurs. 
On constate donc que les travailleuses de la région sont vulnérables à différentes sources de violence dans l'exercice quotidien de leur métier. Nos données reconduisent un des constats de la revue de littérature : les travailleuses du sexe de rue ont des besoins impératifs en matière de sécurité. Les sources de risque sont multiples comme l'indiquent systématiquement des représentantes des travailleuses du sexe au pays et ailleurs dans le monde et comme l'a constaté récemment le Ministère de la Justice du Canada (1998) qui s'est penché sur le travail du sexe de rue au Canada. Les réponses proposées, nous l'avons indiqué, varient selon la conception que l'on a du travail du sexe.

\section{La santé des travailleuses}

Comme nous l'avons indiqué précédemment, l'ensemble des recherches met en évidence que les travailleuses du sexe de rue consomment des drogues et de l'alcool dans un pourcentage important et nettement plus élevé que les autres catégories de travailleuses du sexe. Dans notre échantillon, toutes les participantes ont fait état de problèmes à ce sujet pendant une période de leur vie. Au moment des entrevues, six d'entre elles travaillaient sans consommer de drogues ou d'alcool, sept consommaient des drogues tout en travaillant, et enfin, six autres ne consommaient plus et avaient quitté le travail du sexe de rue.

Par contre, nos données infirment, encore une fois, l'association automatique entre le travail du sexe et les infections transmises sexuellement. Dans notre échantillon, quatre travailleuses rapportent des problèmes sur ce plan. L'une a contracté le VIH et l'hépatite C, une autre a le SIDA et éprouve des problèmes de santé mentale. Les deux autres souffrent d'hépatite C.

Dans l'ensemble, les travailleuses ne rapportent pas de problèmes de santé physique, mais deux d'entre elles éprouvent des problèmes de santé mentale.

\section{Les relations sociales}

Le choix de ce type de travail en association avec la consommation de drogues entraîne des problèmes au niveau des relations sociales. 
Nos données indiquent que les travailleuses du sexe de notre échantillon constituent un groupe marginalisé jusque dans les relations non officielles et intimes, au niveau de la famille et des amis. Plusieurs vivent dans l'isolement. Cette donnée n'est pas étonnante, mais elle est peu mise en évidence dans nombre de recherches sur les besoins quotidiens des travailleuses du sexe de rue. Il nous apparait important de la mettre de l'avant lorsque nous envisageons la mise sur pied de services pour répondre à leurs besoins.

En effet, nous avons constaté que, dans l'ensemble, le choix d'exercer cette forme de travail qui, contrairement à d'autres formes de travail du sexe, ne semble pas pouvoir être cachée à ses relations proches, cause généralement des problèmes. Dans certains cas, les relations avec la famille sont plutôt froides, hostiles, dans d'autres les liens sont brisés. L'expérience de Julie est révélatrice à cet égard :

"Ma famille ne m'a pas parlé. Ma grand-mère est décédée quatre ans passés, elle m'a dit qu'elle m'aimait, mais pendant le sept, huit ans que j'étais dans la prostitution elle ne me parlait plus. Puis ma søeur non plus, elle me parle quasiment plus. Ils sont trop déçus. (...). Mon grand-père c'est l'amour inconditionnel. Mais le reste de ma famille me parle quasiment pas. »

Lorsqu'elles décident de cesser de consommer des drogues ou de quitter ce secteur de travail, quelques femmes réussissent à rétablir les liens avec des membres de leur famille. Cocotte a été rejetée par ses sœurs, son père et l'ensemble de sa parenté lorsqu'ils ont appris qu'elle était travailleuse du sexe de rue. Mais, depuis qu'elle a quitté le métier, ils ont repris contact avec elle : «Asteure, ma famille vient toute me voir puis ils viennent chez nous, ma soeur vient chez nous, elle vient me parler. Ma mère vient me parler, mon père aussi. C'est tout arrangé parce qu'ils me parlent. Ils font pas mal le tour".

Notons, par ailleurs, que la majorité des femmes rencontrées vivent seules, sans partenaires et sans enfants. Plusieurs ont perdu la garde de leurs enfants, mais six d'entre elles ont un ou une partenaire et quatre d'entre elles habitent en couple. 
"L'isolement social devient encore plus évident lorsque les femmes nous parlent de leurs relations d'amitié. Les travailleuses du sexe franco-ontariennes sont particulièrement esseulées. "
L'isolement social devient encore plus évident lorsque les femmes nous parlent de leurs relations d'amitié. Les travailleuses du sexe franco-ontariennes sont particulièrement esseulées. Deux d'entre elles, Brigitte et Samantha, affirment qu'elles n'ont pas d'amis, de confidents, que leur seul soutien est celui d'intervenantes en qui elles ont pleinement confiance. Par contre, cinq des six travailleuses du sexe de rue anglophones rapportent pouvoir s'appuyer sur des amis. Une seule compte sur des professionnelles pour un soutien psychologique. Au Québec, quatre femmes sur six, qui par ailleurs bénéficient d'un appui professionnel important, peuvent compter sur des amis. Daphnée affirme en effet : "Madeleine là, puis moi là, je la considère pas comme une travailleuse de rue, je la considère comme une sœur que j'ai jamais eue ».

\section{Le logement}

De façon générale, le logement constitue un problème pour les femmes qui exercent le métier dans la rue. La revue de littérature en fait état. Par ailleurs, dans les régions d'Ottawa et de Gatineau, le taux de vacance des logements locatifs est extrêmement faible depuis nombre d'années, de sorte qu'à Gatineau, tous les $1^{\text {er }}$ juillet, lors du renouvellement des baux en Outaouais, nombre de familles socioéconomiquement défavorisées se retrouvent sans logement. Les municipalités doivent intervenir pour les aider à se reloger. On peut comprendre que dans cette conjoncture, les travailleuses du sexe de rue qui, pour plusieurs, n'ont pas une vie très stable et qui font aisément l'objet de stigmatisation, éprouvent d'énormes problèmes à trouver et à conserver un logement adéquat et sécuritaire.

Au moment des entrevues, durant l'été 2004, 16 des 19 participantes rapportent avoir des problèmes de différents ordres à ce sujet. Pour six d'entre elles, le problème est tel qu'elles sont tout simplement sans logement. Elles habitent dans des refuges pour femmes ou encore chez un membre de la famille (chez les parents ou chez un des enfants). Quelques-unes louent une chambre, mais à prix fort et ne bénéficient pas de l'espace, ni de la sécurité que peut procurer un logement. Plusieurs, en chambre ou en logement, 
éprouvent des problèmes sérieux, soit avec le propriétaire qui veut les chasser, soit avec des colocataires ou encore avec des voisins qui leur rendent la vie difficile. Il semble que leur choix de travail, associé pour un certain nombre d'entre elles à la consommation de drogues, constitue une source de conflits avec l'entourage et rend plus difficile la quête d'un logement stable et sécuritaire.

\section{Recours aux services disponibles}

Dans l'ensemble, les participantes ont indiqué une série de besoins en matière de services. Par ailleurs, nous avons constaté qu'elles connaissent bien toute une panoplie de ressources et se prévalent de leurs services lorsque nécessaire. Elles fréquentent quelquesunes d'entre elles sur une base très régulière. Une forte majorité des travailleuses, soit 14 , affirment avoir recours aux banques de nourriture; un même nombre fréquente les centres de jour; il semble que presque toutes celles qui utilisent les banques de nourriture ont recours aux centres de jour. Nous avons également observé que 18 participantes utilisent des services de santé et huit d'entre elles des ressources en toxicomanie.

Donc, on constate qu'en général, les femmes utilisent plusieurs des services disponibles, bien que la liste varie d'une femme à l'autre. Par exemple, au Québec, les femmes réferent à des organismes comme le CLSC, Gîte-Ami, Bras, CIPTO, le Pavillon Jelinek, la Société St-Vincent de Paul, l'entraide familiale, la Soupe populaire de Hull, etc.; du côté ontarien, les participantes utilisent tour à tour le Sandy Hill Community Centre Outreach, le Sexual Health Center, Site Van, Centre 454, Salvation Army, OASIS, le Centre Espoir Sophie, etc.

Dans l'une ou l'autre des ressources, bien qu'elles n'aient pas toutes recours aux mêmes services, toutes les femmes trouvent un appui, un soutien psychologique ou émotif, auprès d'un intervenant ou d'un employé. Il est intéressant et rassurant de constater que les participantes peuvent y trouver non seulement un appui matériel, mais aussi le soutien d'intervenantes qui établissent 
"...les participantes peuvent $y$ trouver non seulement un appui matériel, mais aussi le soutien d'intervenantes qui établissent avec elles des relations significatives qui vont souvent bien au-delà de leur strict mandat professionnel. Ainsi, pour les femmes qui ont peu ou pas de relations sociales, ces contacts constituent des pôles de référence importants dans leur quotidien." avec elles des relations significatives qui vont souvent bien au-delà de leur strict mandat professionnel. Ainsi, pour les femmes qui ont peu ou pas de relations sociales, ces contacts constituent des pôles de référence importants dans leur quotidien.

Remarquons, par ailleurs, que si les participantes utilisent un bon nombre de ressources, celles-ci sont, dans une large mesure, destinées à répondre aux besoins de groupes marginalisés de la population, comme le Gîte-Ami, la Société St-Vincent de Paul, la Soupe populaire de Hull, Sherpherds of Good Hope, Salvation Army, The Mission, St-Joe, etc. Elles ont aussi recours à des services où elles se sentent bien accueillies, comme le Site Van, Sexual Health Centre, le Bureau régional d'action SIDA (BRAS), etc. Mais elles font peu souvent référence et semblent ne pas utiliser nombre de services destinés à la population en général, comme des services éducatifs, de loisirs, des programmes généraux de formation à l'emploi ou de recherche d'emploi.

En somme, les travailleuses de sexe de rue de la région se débrouillent bien pour trouver réponse à leurs besoins, du moins dans une certaine mesure. Elles ciblent les ressources qui peuvent, à la fois, répondre à leurs besoins et le faire sans porter un jugement moral négatif sur elles. Elles cherchent ainsi à se protéger de la stigmatisation dont elles font trop souvent l'objet dans la population. Cette capacité est particulièrement mise en évidence lorsqu'on constate que, malgré l'absence de services de soutien psychologique pensés pour elles, ces femmes trouvent un appui chez des intervenantes dans les services déjà en place.

Par contre, leurs besoins sont nombreux et on note l'absence de ressources dont le mandat est de répondre à un ensemble d'entre eux, comme certains centres de jours dont nous avons fait état dans la revue de littérature. On peut également mesurer le vide en matière de coordination de services malgré les besoins multiples de ce groupe de femmes et malgré la dispersion géographique de cette clientèle sur deux juridictions provinciales et plusieurs territoires de travail. 


\section{Les services nécessaires... voire de rêve}

Comment répondre à ces besoins multiples? Nous l'avons demandé aux participantes qui ont énuméré une série de services à mettre en place, sans toutefois les hiérarchiser ou mettre en évidence les besoins de coordination entre ceux-ci. Cela n'a rien d'étonnant puisqu'elles ne sont ni des responsables de politiques ni des intervenants dont le mandat est d'évaluer l'ensemble des besoins d'une clientèle et les stratégies les plus adéquates pour y répondre. Elles sont confrontées dans le quotidien à une panoplie de problèmes et, pour elles, la solution des uns n'atténue pas l'urgence d'en résoudre nombre d'autres. Elles font état, tour à tour, de besoins qu'elles partagent avec d'autres groupes marginalisés de la région, de besoins de changements en matière de politiques criminelles et de besoins en programmes spécifiques qui rendent compte de la configuration unique de leurs problèmes.

Remarquons d'abord que quatre participantes réclament des services en matière de logement. Avec d'autres groupes marginalisés, elles sont confrontées à la pénurie d'appartements qui frappe la région, mais leur style de vie et la stigmatisation dont elles font l'objet nous portent à croire qu'on doit leur apporter une attention particulière et mettre en place des programmes ciblés, comme les maisons d'hébergement pour les travailleuses du sexe que proposent Thukral et Ditmore (2003) à New York. Cette mesure permettrait à des femmes d'instaurer plus de stabilité dans leur quotidien et d'améliorer leur vie.

Certaines ont souligné les besoins d'appui des travailleuses en matière d'éducation soit pour retourner à l'école, soit pour entreprendre des démarches de recherche d'emploi. Elles auraient besoin de conseils, d'information, d'assistance dans leurs démarches. Deux participantes en ont fait spécifiquement état. Smiley a pointé du doigt le manque de ressources pour y répondre à la suite des compressions du gouvernement provincial ontarien en matière de programmes sociaux.

Qui plus est, compte tenu de la marginalisation des travailleuses du sexe de rue et des problèmes de santé rencontrés, certaines 
"...il ressort

clairement qu'elles voudraient bien travailler en toute sécurité sans se soucier d'enfreindre constamment des lois et en bénéficiant de la même protection que toutes les autres citoyennes. Du même souffle, elles demandent une meilleure protection policière." d'entre elles doivent, par moments, recourir à l'assistance sociale; comme tous les autres bénéficiaires de ce service, elles constatent que les sommes versées sont tout à fait insuffisantes pour répondre à leurs besoins de base. Aussi, une des participantes signale la nécessité de hausser ces prestations.

Face à leurs besoins psychologiques, on a vu que les participantes trouvent appui auprès de leurs relations proches ou auprès d'intervenantes. Mais les services psychologiques qui sont déjà fort limités pour la population apparaissent fort lacunaires. À cet effet, une des participantes souhaite qu'on accroisse les options offertes aux femmes.

Si tout justiciable confronté à la justice a besoin des services d'un avocat ou d'une avocate, les travailleuses du sexe de rue, dont certaines activités de travail sont criminalisées, doivent, d'une manière générale, s'en prévaloir plus fréquemment. Une des participantes a évoqué le besoin de pouvoir compter sur l'appui d'une professionnelle sympathique, prête à écouter sans juger.

En ce qui concerne les politiques criminelles, c'est sans surprise qu'on a constaté que les femmes favorisent la décriminalisation ou la réglementation de leurs activités. Elles ne précisent pas exactement la formule à privilégier, mais cela n'est pas étonnant. En effet, même les experts en la matière ne s'entendent pas sur les paramètres de chaque formule et les débats et discussions font toujours rage sur cette question. Par contre, il ressort clairement qu'elles voudraient bien travailler en toute sécurité sans se soucier d'enfreindre constamment des lois et en bénéficiant de la même protection que toutes les autres citoyennes. Du même souffle, elles demandent une meilleure protection policière.

Mais, pour l'instant, comme elles constituent un groupe fort vulnérable à la violence de différentes sources, dont celle des clients, quatre travailleuses ont mis de l'avant le besoin de créer une liste de mauvais clients. Cette mesure peut être fort précieuse pour les femmes si la liste est systématiquement tenue à jour et mise à portée de mains de toutes.

En matière de services spécifiques, 12 participantes demandent un centre de jour. Elles disent explicitement qu'elles veulent un 
lieu où elles ne seront pas jugées, où elles pourraient bénéficier de services de santé, de soutien psychologique, et qui demeurerait ouvert le soir et la nuit jusqu'à trois ou quatre heures du matin. Cette demande reflète la voix d'une grande majorité des femmes. Certaines réferent également à cet endroit comme à un lieu qui leur permettrait de briser l'isolement, de socialiser. Julie souligne les besoins auxquels un centre de jour pourrait répondre : "qu'elles aient une place juste de filles là (...) Une place pour se changer, pour s'habiller, pour parler aussi ». Samantha précise : " un endroit où il y a du café, des beignes, il y a des pauses café; où on trouve peut-être sur place deux ou trois intervenants puis qui ont différentes compétences pour enligner les femmes. ».D'emblée, les participantes identifient les centres de jour comme des ressources aptes à répondre à un ensemble de leurs besoins. La revue de littérature a permis de constater que ces ressources, lorsque bien pensées pour les travailleuses du sexe, sont fort fréquentées. Mais, on doit reconnaitre qu'elles ne peuvent être mises en œuvre sans un soutien financier adéquat et sans s'assurer la collaboration de plusieurs professionnels spécialisés dans différents services psychologiques, sociaux et de santé. Dans la région d'OttawaGatineau, ce ou ces centres de jour devraient être localisés de façon à répondre à une clientèle qui, rappelons-le, est dispersée sur un territoire assez vaste. Pour assurer un programme adapté, nous pourrions, par exemple, mettre sur pied deux centres de jour qui offriraient un éventail similaire de services, mais se partageraient certaines ressources spécialisées.

Parmi les participantes, sept ont manifesté le besoin d'un lieu pour travailler. Pour certaines, un quartier réservé pour le travail du sexe leur assurerait plus de sécurité; pour d'autres, un établissement de travail apparait comme une bonne solution. Smiley favorise la mise en place de maisons de travail afin de protéger les femmes des agressions.

Deux travailleuses du sexe souhaitent la mise sur pied de services mobiles pour rencontrer les travailleuses du sexe, comme des travailleuses qui se déplacent en voiture pour offrir des services médicaux, d'hygiène corporelle ou encore, une roulotte mobile qui serait ouverte de 22 heures à 8 heures. 
Nous avons demandé à 15 des travailleuses si elles étaient pour la mise sur pied d'une organisation pour travailleuses du sexe dans la région. Toutes ont répondu par l'affirmative, bien qu'aucune n'ait évoqué par elle-même ce besoin de regroupement. Cette option s'est pourtant avérée fort positive pour les travailleuses du sexe de certaines villes du pays et il nous apparaît opportun de pousser plus loin la recherche à ce sujet.

\section{Conclusion}

«En somme, notre recherche confirme que les travailleuses du sexe de rue, comme leurs collègues qui ouvrent dans d'autres secteurs de l'industrie du sexe, se définissent en grande majorité comme des travailleuses. Elles demandent donc la reconnaissance de leurs droits, elles réclament la décriminalisation de leurs activités. »
En somme, notre recherche confirme que les travailleuses du sexe de rue, comme leurs collègues qui œuvrent dans d'autres secteurs de l'industrie du sexe, se définissent en grande majorité comme des travailleuses. Elles demandent donc la reconnaissance de leurs droits, elles réclament la décriminalisation de leurs activités. Les entrevues ont permis de mettre en évidence le fait que le contrôle pénal en place les marginalise et les rend vulnérable à la violence et à l'exploitation. Nombre de leurs besoins actuels (logement, services juridiques, protection contre la violence) sont directement reliés au cadre juridique auquel elles sont soumises. Par ailleurs, il faut reconnaître que la décriminalisation, qui nous apparait essentielle, ne constitue pas une panacée pour les travailleuses du sexe de rue. Certains de leurs besoins sont associés à la stigmatisation sociale dont elles font l'objet, qu'il serait certes plus facile de contrer dans un contexte de décriminalisation, mais qui ne disparaitrait pas pour autant. Des initiatives comme les centres de jour pourraient s'avérer encore tout aussi importantes. Il faudrait, en outre, mettre en œuvre des mesures pour favoriser la reconnaissance du travail du sexe comme une forme de travail dans le domaine des services. Enfin, pour certaines de ces femmes qui ont des problèmes de toxicomanie, la réponse à leurs besoins nous apparait passer d'abord par la mise en place de solutions sur ce plan. Mais, s'il n'y a pas de solution unique pour résoudre les problèmes rencontrés par les travailleuses du sexe de rue, il ne faut pas pour autant renoncer à la décriminalisation qui nous 
apparait encore et toujours comme une condition préalable pour améliorer les conditions de travail de ces femmes de même que leur existence.

\section{Bibliographie}

BENOIT, Cecilia et Alison MILLAR (2001). Dispelling Myths and Understanding Realities: Working Conditions, Health Status, and Exiting Experiences of Sex Workers, www.peers.bc.ca/pubs.html

BENSON, Catherine et Roger MATTHEWS (1995). «Street Prostitution:Ten Facts in Search of a Policy», International Journal of the Sociology of Law, vol. 23, 395-415.

BLOM, Maria et Ton van den BERG (1989). «A Typology of the life and Work Styles of 'HeroinProstitutes'», dans Maureen Cain ed. Growing up Good. Policing the Behaviour of Girls in Europe, London, Sage, 55-69.

BOYLE, Kathleen et M. Douglas ANGLIN (1993), "To the Curb’ : Sex Bartering and Drug Use among Homeless Crack Users in Los Angeles» dans Mitchell S. Ratner ed. Crack Pipe as a Pimp, New York, Lexington Books, 159-186.

BRUCKERT, Chris, Colette PARENT et Pascale ROBITAILLE (2003). Établissements de services érotiques/danse érotique : deux formes de travail marginalisé, Ottawa, Commission du Droit du Canada.

BULLOUGH, Bonnie etVern L. BULLOUGH (1998). «Introduction. Female Prostitution : Current Research and Changing Interpretations", dans James Elias,Vern Bullough et al. eds. Prostitution. On Whores, Hustlers, and Johns, Amherst, Prometheus Books, 158-180.

DALLA, Rochelle, L. (2002). "Night Moves: A Qualitative Investigation of Street-Level SexWork”, Psychology of Women Quarterly, vol. 26, 63-73.

GEMME, Robert (1998). "Legal and Sexological Aspects of Adult Street Prostitution: a Case for

Sexual Pluralism”, dans James Elias, Vern Bullough et al. eds. Prostitution. On Whores, Hustlers, and Johns, Amherst, Prometheus Books, 474-487.

GIBSON, Mary (1993). «Italy» dans Nanette J. Davis ed. Prostitution: An International Handbook on Trends, Problems, and Policies, London, Greenwood Press.

GUÉNETTE, José et Michelle LÉVEILLÉ (2002). Au pays des dames de nuit. Document préparé à l'intention du Centre d'intervention et de prévention en toxicomanie de l'Outaouais, CIPTO, mai.

INCIARDI James A., Dorothy LOCKWOOD et Anne E. POTTIEGER (1993). Women and CrackCocaine, New York, Macmillan.

INTERNATIONAL COMMITTEE FOR PROSTITUTES RIGHTS (1985). World Charter for Prostitutes' Rights, http://www.walnet.org/csis/groups/icpr_charter.html.

KOESTER, Stephen et Judith SCHWARTZ (1993). «Crack, Gangs, Sex, and Powerlessness: a View from Denver» dans Mitchell S. Ratner ed. Crack Pipe as a Pimp, New York, Lexington Books, 187-204.

LOCKHART, Chris (2002). «Kunyenga, "Real Sex”, and Survival:Assessing the Risk of HIV Infection among Urban Street Boys in Tanzania», Medical Anthropology Quarterly, vol.16, no. 3, 294-311.

LOWMAN, John et Laura FRASER (1995). Violence against Persons Who Prostitutes: The Experience of British Columbia, Ottawa, Ministère de la Justice. 
LOWMAN, John (1985-86). "Prostitution in Canada», Resources for feminist Research/Documentation sur la Recherche Féministe, vol.14, no. 4, Dec-Jan, 35-37.

McKEGANEY Neil et Marina BARNARD (1996). Sex Work on the Streets: Prostitutes and their Clients, Buckingham, Open University Press.

MILLER, Eleanor, Kim ROMENESKO et Lisa WONDOLKOWSKI, (1993). «The United States», dans Nanette J. Davis ed. Prostitution: An International Handbook on Trends, Problems, and Policies, London, Greenwood Press, 300-326.

Ministère de la Justice du Canada (1998). Rapport et recommandations relatives à la législation, aux politiques et aux pratiques concernant les activités liées à la prostitution, Groupe de travail fédéral/provincial/territorial sur la prostitution, http://canada.justice.gc.ca/fr/news/nr/1998/toc.html

NORTON-HAWK, Maureen A. (2001). «The Counterproductivity of Incarcerating Female Street Prostitutes», Deviant Behavior:An Interdisciplinary Journal, vol. 22, 403-417.

NSWP (2004). Sex workers' Manifesto, http://www.walnet.org/csis/nswp/conferences/manifesto/ html

PAENT, Colette et Chris BRUCKERT (2005). « Le travail du sexe dans les établissements de services érotiques : une forme de travail marginalisé ", Déviance et Société, vol. 29, no. 1,33-54.

PHETERSON, Gail ed (1989). A Vindication of the Rights of Whores, Seattle, Seal Press.

PRESTON-WHITE, E (1996). «Survival Sex or the Culture of Sex Work in South Africa» AIDS Bulletin, vol.5, no.1, 8-9.

PRYEN, Stéphanie (1999). Stigmate et métier. Une approche sociologique de la prostitution de rue, Rennes, Presses Universitaires de Rennes.

PYETT, Priscilla et Deborah WARR (1999). «Women at Risk in Sex Work: Strategies for Survival», Journal of Sociology, vol. 35, no. 2, August, 183-197.

RABINOVITCH, Jannet et Susan Strega (2004). «The PEERS Story», Violence Against Women, vol. 10, no. 2, February, 140-159.

RATNER, Mitchell S. (1993). «Sex, drugs and Policy: Studying and Understanding the Sex-forCrack Phenomenon", dans Mitchell S. Ratner ed. Crack Pipe as a Pimp, New York, Lexington Books, 1-36.

RICHTER Linda M. et Jill SWART-KRUGER (1995). "AIDS-risk among Street Children and Youth : Implications for Intervention", South African Journal of Psychology, vol.25, no.1, 31-38.

ROSENBAUM, Marsha (1981). Women on Heroin. New Brunswick, New Jersey, Rutgers University Press.

SPITTAL, Patricia M., J BRUNEAU, K.J.P. CRAIB, C. MILLER, F. LAMOTHE, A. E. WEBER, K. LI, M.W TUNDALL, M.V.O'SAUGHNESSY et M.T. SCHECTER (2003). «Surviving the Sex Trade: a Comparison of HIV Risk Behaviours among Street-Involved Women in Two Canadian Cities who Inject Drugs", AIDS Care, vol. 15, no. 2, 187-195.

STERK, Claire E. (1999). Fast Lives. Women Who Use Crack Cocaine, Philadelphia, Temple University Press.

STERK-ELIFSON, Claire, et Carole A. CAMPBELL (1993). «The Netherlands», dans Nanette J. Davis ed. Prostitution: An International Handbook on Trends, Problems, and Policies, London, Greenwood Press, 191-206.

SURRATT, Hilary L., James A. INCIARDI, Steven P. KURTZ, Marion C. KILEY (2004). «Sex Work and Drug Use in a Subculture of Violence», Crime \& Delinquency, vol. 50, no. 1, January, 43-59. 
THUKRAL, Juhu, et Melissa DITMORE (2003). Revolving Door:AnAnalysis of Street Based Prostitution in New York City, NewYork, Urban Justice Center: Sex Workers Project. www.sexworkersprojetct. org/downloads/RevolvingDoor.pdf

TYLER, Kimberley A., Dan R. HOYT et Les B.WHITBECK (2000). «The Effects of Early Sexual Abuse on Later Sexual Victimization among Female Homeless Runaway Adolescents», Journal of Interpersonal Violence, vol.15, no.3, 235-250.

VANWESENBEECK, Ine (1994). Prostitutes Well-Being and Risk. Amsterdam,VU Uitgeverij.

WELZER-Lang, Daniel, Odette BARABOSA et Lilian MATHIEU (1994). Prostitution : les uns, les unes et les autres, Paris, Métaillé.

\section{Notes}

1 Cette recherche a été subventionnée par Condition féminine Canada et la Faculté des sciences sociales de l'Université d'Ottawa.

2 Selon Sterk-Elifson et Campbell (1993 : 200), entre 50 et $80 \%$ des travailleuses du sexe de rue seraient usagères de drogues.

3 Voir le site web de l'organisme : http://perso.wanadoo.fr/cabiria/cabiria.html

4 Voir http://www.drugtext.org/library/articles/peddr0031.htm 